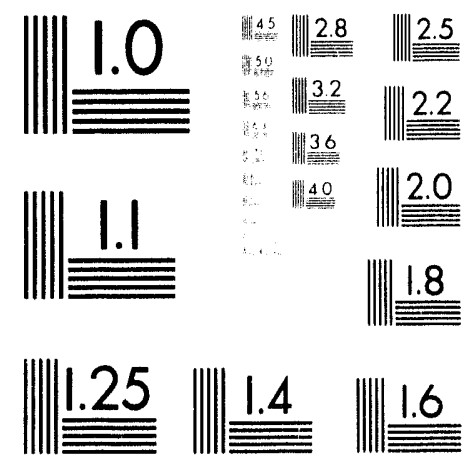



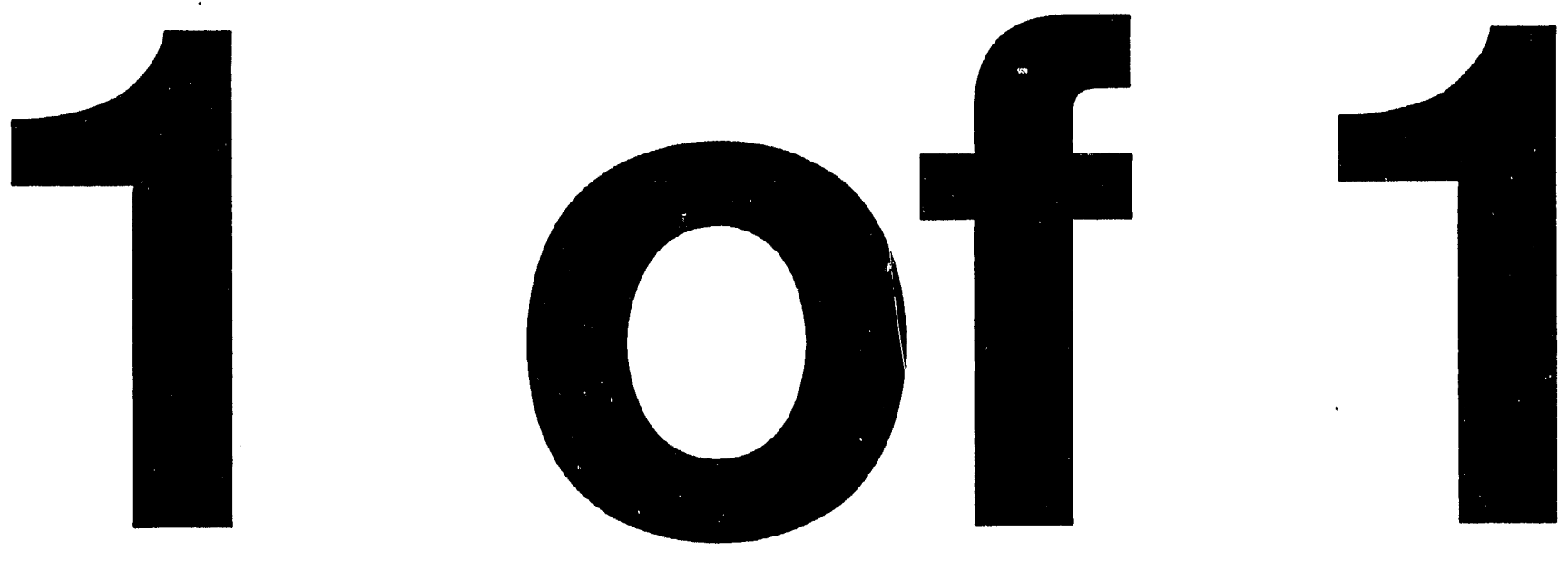
LBL-33242

\title{
A Generalized Entering Coefficient to Characterize Foam Stability Against Oil in Porous Media
}

\author{
V. Bergeron, M. E. Fagan, and C.J. Radke \\ Department of Chemical Engineering \\ University of California \\ and \\ Earth Sciences Division \\ Lawrence Berkeley Laboratory \\ University of California \\ Berkeley, California 94720
}

November 1992

This work was supported by the Assistant Secretary for Fossil Energy, Office of Oil, Shale and Gas Technologies, under U.S. Department of Energy Contract No. DE-AC03-76SF00098.

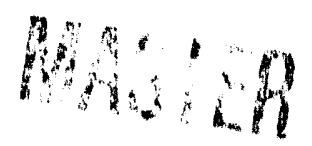




\title{
Symposium on Enhanced Oil Recovery \\ Presented before the Division of Petroleum Chemistry \\ American Chemical Society
}

March 28 - April 2, 1993, Denver Colorado

\begin{abstract}
A Generalized Entering Coefficient to Characterize Foam Stability Against Oil in Porous Media
\end{abstract}

\author{
V. Bergeron, M.E. Fagan, and C.J. Radke \\ Earth Sciences Division of Lawrence Berkeley Laboratory and \\ Department of Chemical Engineering \\ University of California \\ Berkeley, CA 94720 (510) 642-5204
}

\section{Motivation}

Foam as an injected fluid for enhanced oil recovery (EOR) has shown promise, particularly in steamflooding (1-3). In addition, foam is effective as a barrier to gas coning in thin oil zones (4). The unique mobility-control properties (i.e., large flow resistance) of foam in porous media make it an attractive choice. However, even though foam shows potential for improved oil recovery, it is not widely used partly because crude oils destabilize most foams. When flowing foam in porous media coalesces into its two separate phases, liquid and gas, it no longer provides a large flow resistance and is ineffective fur oil recovery. It is imperative for foam to remain stable against oil, that is to remain as a dispersion of gas in liquid, in EOR applications. Therefore, it is important to understand how oil destabilizes 
foam and what surfactant properties lead to increased stability against oil. Figure 1 shows a highly schematic diagram of foam bubbles percolating past a residual oil ganglion in a porous medium (5). The aqueous surfactant solution is indicated by light shading, the rock is represented by cross-hatching, and the oil is shown as dark shading. As a lamella (i.e., a gas-water-gas or foam film) flows by a water-wet sand grain, it deposits a thick water film between the gas and solid. Provided the oil globule is similarly water wet, the flowing lamella can deposit a thick water film in an exactly similar manner on the exposed portion of the oil. The gas-wateroil film that results is coined a pseurloemulsion film (6). It is possible for the pseudoemulsion film to thin and break under the capillary-pressure suction in the Plateau border that terminates the film. In this case the oil may enter the gas-water interface and spread as a gas-oil-water film. Thus, the fate of the deposited pseudoemulsion film is crucial to how oil interacts with foam in porous media; the stability of the pseudoemulsion film is critical to maintain foam stability in the presence of oil.

Recent work on oil destabilization mechanisms of foam focuses on bulk spreading and entering coefficients (7-11), underpinned by the pioneering ideas of Ross (12) and Robinson and Woods (13). For an oil drop arriving at a planar gas-water interface, the classical spreading and entering coefficients are defined from the thermodynamic conditions for the minimization of the surface free energy (14):

Spreading Coefficient:

$$
S_{o w}=\sigma_{w g}-\sigma_{o w}-\sigma_{o g},
$$


Entering Coefficient:

$$
\left.\mathrm{E}_{\mathrm{ow}}=\sigma_{\mathrm{wg}}+\sigma_{\mathrm{ow}}-\sigma_{\mathrm{og}}, \quad 2\right)
$$

where $\sigma_{\mathrm{ij}}$ corresponds to the surface or interfacial tension, and the subscripts $0, \mathrm{w}$, and $\mathrm{g}$ signify oil, water and gas respectively. A positive $\mathrm{S}_{\mathrm{ow}}$ demands oil spreading on a gas-water interface, while a positive $\mathbf{E}_{\mathrm{ow}}$ corresponds to oil penetrating the gas-water interface from the aqueous side. As pointed out by Ross (15) entering and spreading coefficients are related by $E_{o w}=-S_{w o}$. That is, a nonentering oil at the gas-water interface is thermodynamically equivalent to water spreading on a gas-oil interface. Unfortunately, a definitive correlation between oil spreading and foam stability in porous media has not emerged. Further, with Equations 1) and 2) there is no provision to account for any influence of the porous medium on foam tolerance to oil.

Another approach to understanding oil destabilization in porous media concentrates on thin-film stability $(5,6)$. Clearly, the lamellae that comprise a foam must remain stable in order for foam to exist. By observing porelevel events of foam flowing in the presence of oil, Manlowe and Radke (5) concluded that foam stability in contact with oil in an etched-glass porousmedium micromodel is controlled by the stability of the aqueous pseudoemulsion films separating oil and gas, shown in Figure 1. Previously, Kruglyakov (16) showed that the stability of bulk foams also relies on the longevity of asymmetric pseudoemulsion films. Moreover, Kruglyakov asserts that water spreading on oil (i.e., nonentering oil systems) is a necessary, but not sufficient, condition for asymmetrical film stability. This assertion is consistent with the oil tolerance demonstrated by negative entering coefficient systems $(11,13)$. Obviously, the inability of oil to 
penetrate (rupture) the gas-water interface leads to an oil-resistant foam. This situation occurs when the aqueous phase completely "wets" the oil, leaving the foam lamellae unperturbed (c.f., Figure 1).

This work unifies the two approaches presently accounting for oilfoam interactions: spreading behavior and thin-film stability. We demonstrate the correspondences between stable pseudoemulsion films, negative entering coeffients, and oil-tolerant foams. Frumkin-Deryaguin theory is applied to the problem of oil-foam interactions and reveals that stable pseudoemulsion films are essential to maintain oil-tolerant foams. This hypothesis is critically tested by comparing steady-state foam flow behavior in glass beadpacks that contain residual oil, with newly measured, equilibrium disjoining pressure isotherms for both foam and pscudoemulsion films, along with bulk surface and interfacial tensions. Theory

To generalize the ideas of spreading behavior and its relation to foam stability we adopt the theory developed by Frumkin (17) and Deryaguin (18) for wetting fluids on a solid substrate. Recent application of this theory has also been made by Churaev (19) and Hirasaki (20). The underlying principle of the Frumkin-Deryaguin framework is incorporation of thinfilm forces in the description of wetting behavior. These forces, commonly expressed by the disjoining pressure isotherm, account for the stability of thin-liquid films. Inclusion of disjoining pressure into spreading and entering coefficients leads to a more general picture of how entering and spreading relate to film stability, and directly accounts for the influence of the porous medium through the capillary-suction pressure. 
The key step required to include thin-film forces into the classica: form of the spreading or entering coefficient is to adopt the film tension model of the interface (20). Consider a pseudoemulsion film generated by bringing an oil droplet in solution to the gas-water interface. A thin aqueous film first separates the oil from the gas and provides a barrier against oil entering the gas phase. By replacing the oil-gas tension in Equation 2) with the film tension (23) of the aqueous pseudoemulsion film and by expressing the film tension in terms of the disjoining pressure isotherm of that film, a generalized entering coefficient arises $(21,22)$ :

$$
E_{o w}^{g}=-\int_{\Pi\left(h_{\infty}\right)=0}^{\Pi=\Pi(h)} h d \Pi \text {. }
$$

Hirasaki (20) presents an analogous expression for the spreading coefficient of a liquid over a solid substrate. Equation 3) relates the entering (spreading) coefficient to thin-film properties. Since at equilibrium $\mathrm{P}_{c}=\Pi$, we immediately discover the role of the capillary pressure imposed by the porous medium. It determines the upper integration limit in Equation 3). Once this limit is set, the generalized entering coefficient simply becomes the net negative of the area under the disjoining pressure isotherm. In other words, a net positive area defines the system as nonentering. Therefore, surfactant packages that generate pseudoemulsion-film disjoining pressure isotherms with large repulsive branches produce highly stable pseudoemulsion films, and display negative oil-entering behavior and very oil tolerant foams.

Unlike the generalized entering coefficient, the classical entering coefficient has only one value for a given system. It is strictly a function of bulk surface and interfacial tensions and is a subcase of the generalized 
entering coefficient. For our equilibrated surfactant solution/oil/gas systems, the classical entering coefficient closely corresponds to the zero $\mathrm{P}_{\mathrm{c}}$ case of the generalized entering coefficient. The advantage of formulating a general coefficient is that we explicitly include thin-film force barriers and implicitly include the complex nature of the porous media. Here, the capillary pressure imposed on the film is determined by both the geometry and saturation of the phases within the porous medium. Additional ramifications of the differences between the classical and general entering coefficients are detailed elsewhere (21).

To test the ideas presented above we measure and compare the disjoining pressure isotherms of pseudoemulsion and foam films, surface and interfacial tensions, and steady-state pressure gradients of flowing foam in glass beadpacks with and without residual oil. Experimental details are presented elsewhere $(21,22)$. A typical example of the results we obtain follows.

\section{Results}

The effect of residual oil on steady foam flow in porous media and the disjoining pressure isotherms for foam and pseudoemulsion films are shown in Figure 2. Figure 2a graphs disjoining pressure isotherms for dodecane-equilibrated-aqueous sodium-dodecylsulfate-solution ( SDS ) foam films and for equilibrated SDS/dodecane pseudoemulsion films. It also lists the steady-state pressure gradients, $|\Delta \mathrm{P}| / \mathrm{L}$, for the oil-equilibrated SDS solution with and without residual dodecane. The subscripts " $\mathrm{f}$ " and "or" indicate whether the steady-state pressure gradient is for foam or for foam against residual oil, respectively. 
It is clear in Figure 2a that the foam film is considerably more robust than the pseudoemulsion film. The disjoining pressure isotherm for the foam film, shown as open diamonds, is much higher than the pseudoemulsion film disjoining pressure, shown as closed diamonds. The foam-film disjoining-pressure data continue to as high as $\mathrm{P}_{\mathrm{c}}=15 \mathrm{kPa}$, with no rupture observed, which is the limit of our experimental apparatus for this system. The pseudoemulsion-film disjoining pressure in Figure $2 a$ is significantly weaker than the foam film. The pseudoemulsion film ruptures near $0.1 \mathrm{kPa}$, which is several orders of magnitude below the highest disjoining pressure measured for the foam film. The steady pressure gradient of dodecane-equilibrated SDS foam flowing in glass beadpacks is $6.1 \mathrm{MPa} / \mathrm{m}$. With residual oil present the gradient is 0.12 $\mathrm{MPa} / \mathrm{m}$. Clearly, residual oil strongly destabilizes this foam. Thus we find that destabilization in this case correlates directly with the lack of stability of the pseudoemulsion films.

Figure $2 \mathrm{~b}$ shows the disjoining pressure isotherms for Zonyl FSK ( $a$ fluorocarbon surfactant) foam and Zonyl FSK/dodecane pseudoemulsion films. This system is significantly different than the preceding two. Both films show high repulsive disjoining pressures and are stable beyond approximately $1.2 \mathrm{kPa}$. Neither film ruptures before we reach the highest obtainable pressures for this system. Both films evidence highly stable repulsive disjoining pressures. The steady pressure gradient for Zonyl FSK against $29 \%$ residual dodecane is $22 \mathrm{MPz} / \mathrm{m}$ versus $19 \mathrm{MPa} / \mathrm{m}$ without residual oil or oil equilibration. Along with the disjoining pressure isotherms, this means that dodecarie does not destabilize Zonyl FSK. Again 
the stability of the foam correlates with the stability of the pseudoemulsion films.

\section{Conclusions}

Figures $2 a$ and $2 b$ together with similar data on a wide variety of systems (21) lead us to conclude that highly repulsive pseudoemulsion film disjoining pressure isotherms (i.e., stable pseudoemulsion films) produce negative generalized entering coefficients and oil-tolerant foams. This in turn provides us with a surfactant design criterion needed to produce oiltolerant foam in porous media.

\section{Acknowledgements}

This work was partially supported by the U.S. Department of Energy under Contract DE-AC03-76SF00098 to the Lawrence Berkeley Laboratory of the University of California.

\section{References}

[1] Holm, L.W., JPT, Vol. 22, No. 12, 1499-1506 (1970).

[2] Mohammadi, S.S., Slyke, D.C., and Ganong, B., SPERE, Vol.4, No. 1, 716 (1989).

[3] Patzek, T.J., and Koinis, M.T., JPT, Vol. 42, No. 4, 496-503 (1990).

[4] Hanssen, J.E., Rolfsvåg, T.A., Corneliussen, R., and Dalland, M., paper presented at $5^{\text {th }}$ European Symposium on Improved Oil Recovery, Budapest, Hungary, (1989).

[5] Manlowe, D.J., and Radke, C. J., SPERE, Vol. 5, No. 4, 495-502 (1990).

[6] Nikolov, A.D., Wasan, D.T., Huang, D.D., and Edwards, D.A., SPE paper 15443, presented at 61st Annual Tech. and Exhibition of the SPE, New Orleans, LA, 1-16 (1986). 
[7] Lau, H.C., and O'Brien, S.M.,SPERE, Vol. 3, No.3, 893-896 (1988).

[8] Kristiansen, T.S., and Holt, T.,preprint, SPE $8^{\text {th }}$ Symp. on Fnhanced Oil Recovery, Tulsa, Oklahoma, April 22-24, SPE paper 24182, 2'‘9-287 (1992).

[9] Holt, T., and Kristiansen, T.S., Summaries of papers for the SPOR-

Seminar, NPD, Stavanger, 59-77 (1990).

[10] Kuhlman, M.I., JPT, Vol. 42, No. 7, 902-908 (1990).

[11] Schramm, L.L., and Novosad, J.J., Colloids and Surfaces, Vol. 46, 21$43(1990)$.

[12] Ross, S., Chem. Eng. Prog., Vol. 54, 429-436 (1950).

[13] Robinson, J.V., and Woods, W.W., J. Soc. Chem. Ind., Vol. 67, 361-364 (1948).

[14] Harkins, W.D., J. Chem. Physics, Vol. 9, 552- 568 (1941).

[15] Ross,S., Chem. Eng. Prog. Vol. 63,No.9,41,1967.

[16] Kruglyakov, P.A., in Thin Liquid Films - Fundamentals and

Applications, ed. Ivanov, I.B., Marcel Dekker Inc., New York, Chapter 11, 767-827 (1988).

[17] Frumkin, A.N., Zh. Fiz. Khim. (1938) Vol 12, 337-345, 1938.

[18] Derjaguin, B.V., Zh. Fiz. Khim. Vol 14, 137-147, 1940.

[19] Churaev, N.V.,Revue. Phys. Appl. 23, 975-987, 1988.

[20] Hirasaki, G.J., in Interfacial Phenomena in Petroleum Recovery, ed. Morrow, N.R., Marcel Dekker Inc., New York, Chapter 2, 23-76 (1991).

[21] Fagan, M.E., M.S. Thesis, University of California, Berkeley (1992).

[22] Bergeron, V., Ph.D. Thesis, University of California, Berkeley (in preparation)

[23] de Feijter, J., in Thin Liquid Films - Fundamentals and Applications, ed. Ivanov, I.B., Marcel Dekker Inc., New York, Chapter 1, 1.47 (1988). 


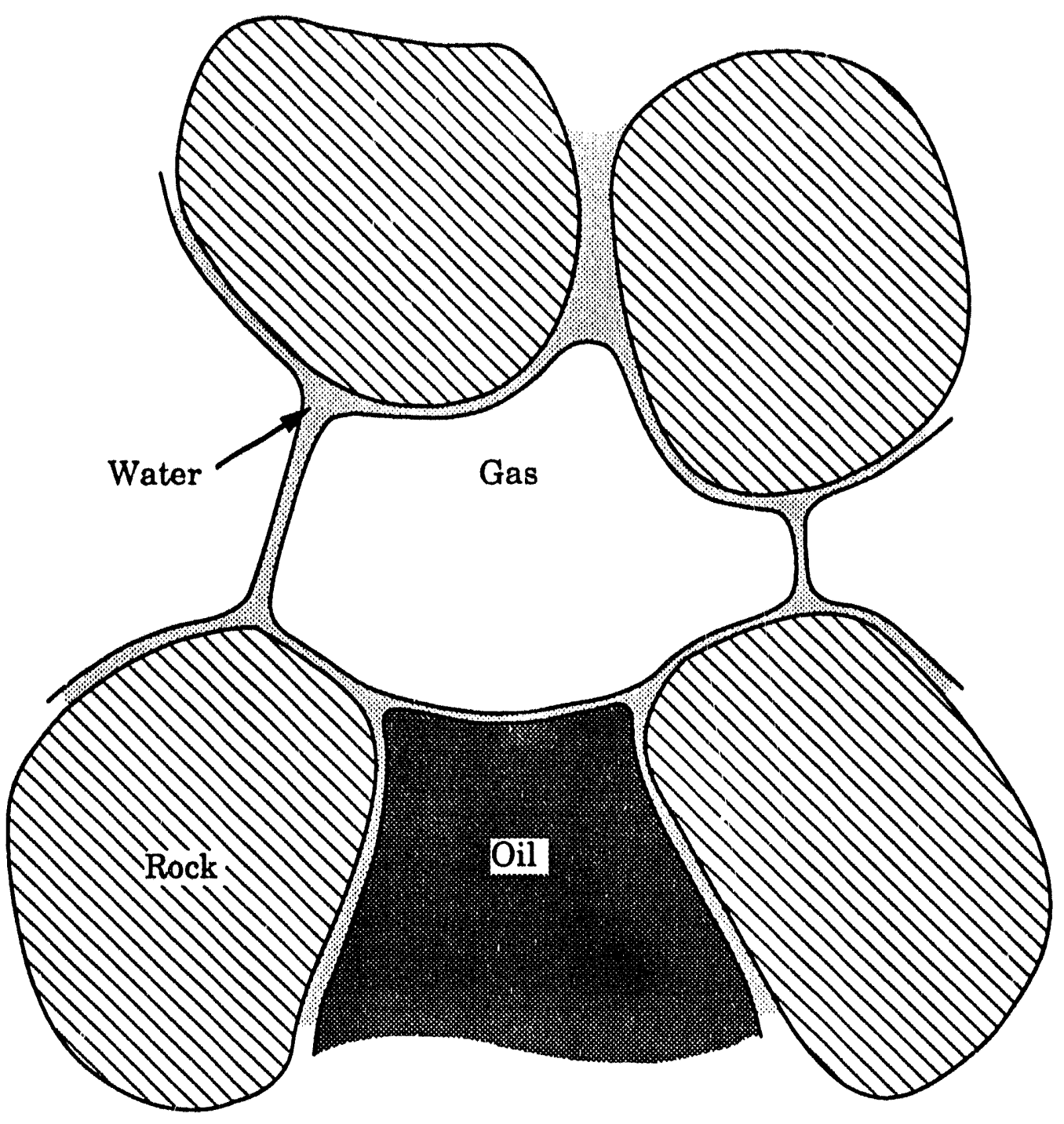

Figure 1. A schematic of foam flowing through an oil reservoir at residual oil saturation (after Manlowe and Radke [5]). 


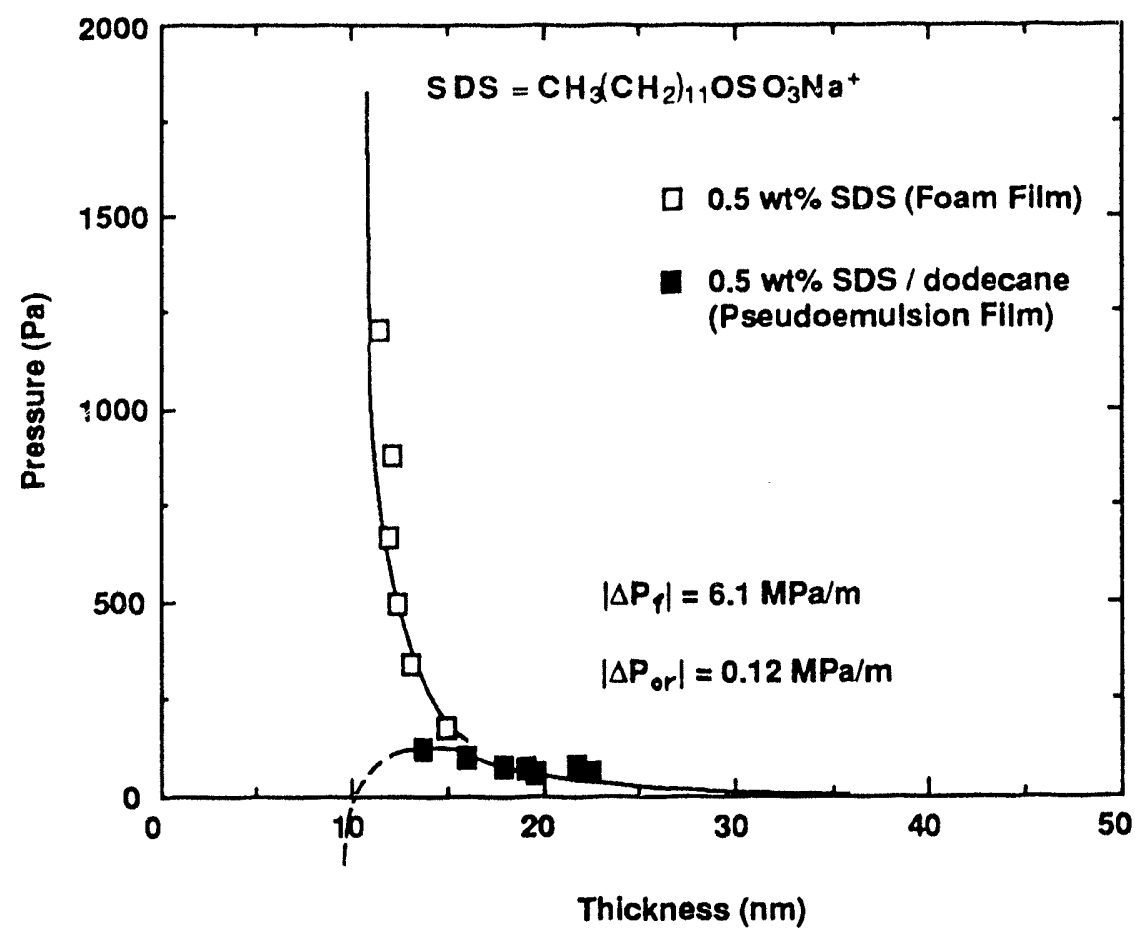

Figure 2a. Foam and pseudoemulsion film disjoining pressures for an oil intolerant system.

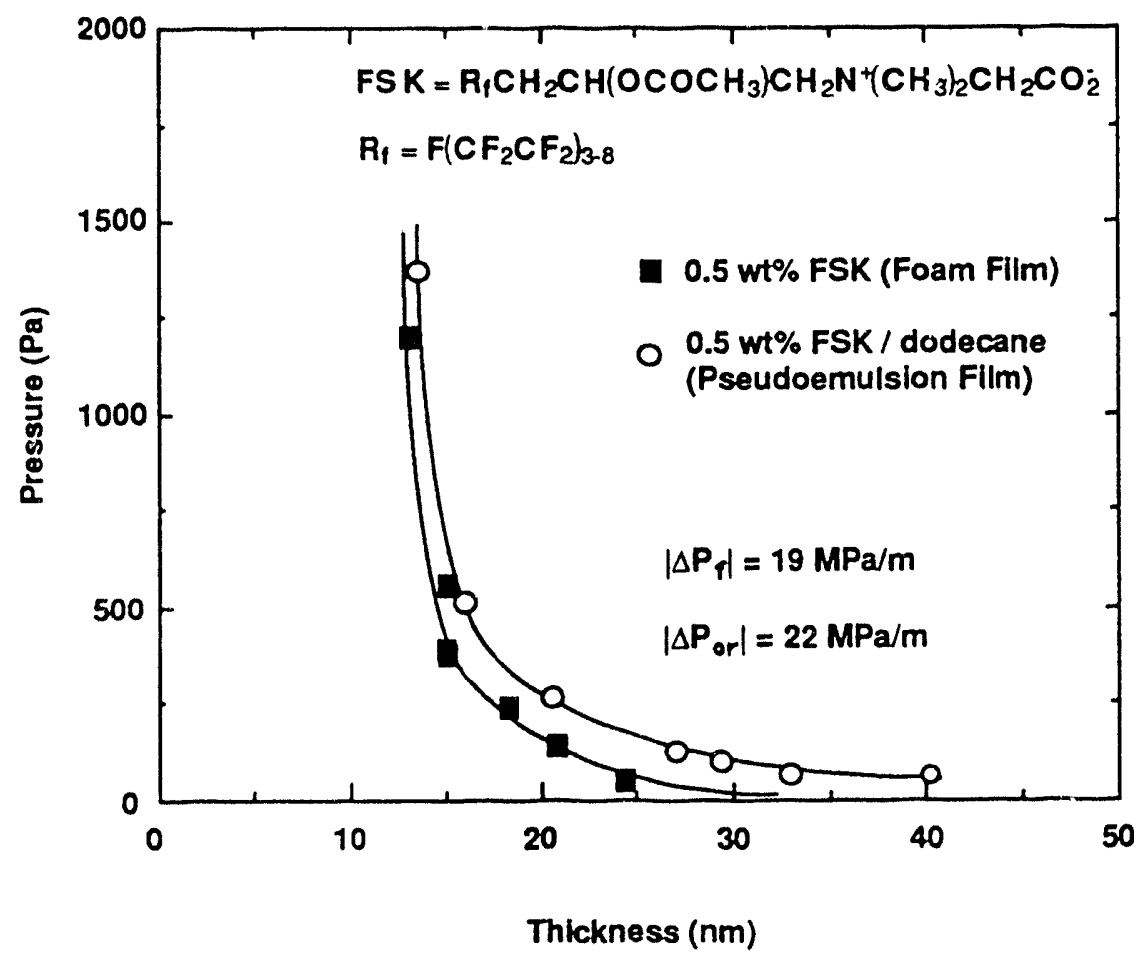

Figure 2b. Foam and pseudoemulsion film disjoining pressures for an oil tolerant system. 

